care and lack of specialist face-to-face support often resulted in unnecessary/unwanted admissions to hospital.

Aims To question the need for an out-of-hours visiting service and to develop the future planning of services to support people at the end-of-life to die at home.

Method Three-year funding was secured, and the service was introduced in 2019. The service provides a clinical nurse specialist and healthcare assistant out-of-hours to deal with calls from known and unknown patients in our communities. The service offers telephone advice, face-to-face consultations and accepts new referrals for people often in crisis. The service works in collaboration with London Ambulance Service (LAS), out-of-hours general practitioners and district nurses.

Results Joint working with LAS developed an appropriate care pathway for all end-of-life patients in the three boroughs. Most calls into the service are for symptom management and emotional support, however, new patient referrals have increased with the team often seeing people in crisis. The activity of the service has increased significantly from year 1 to year 3 with $50 \%$ of calls from patients and $50 \%$ from professionals.

Conclusion The benefits of the service are evident in the data reports and the patient experience feedback, it has enhanced the community service by ensuring a seamless service for people at the end-of-life and supporting next of kin and professionals 24/7. The service has prevented admissions to hospital reducing the pressure on the acute sector. The data provides evidence for the need of $24 / 7$ visiting and we are currently exploring funding options to continue the service.

\section{P-123 THE ROLE OF A PARAMEDIC IN A COMMUNITY HOSPICE/PALLIATIVE/END-OF-LIFE CARE TEAM}

Michael Singer. Mountbatten Hospice, Newport, UK

\subsection{6/spcare-2021-Hospice.140}

Introduction The evolution of the paramedic role over recent years has changed dramatically, with an increased emphasis on treating more patients in the community rather than conveying to hospitals unnecessarily. Paramedics see and treat a diverse range of patients from acute events to chronic conditions, many patients have co-morbidities and polypharmacy in an aging population. Thus, the modern paramedic must be equipped with holistic clinical skills over a wide variety of specialties.

Multi-disciplinary working in hospices is well established but does not typically include paramedicine. Paramedicine has changed in recent years extending beyond the typical emergency response model to encompass community paramedicine with paramedics working in primary care but the role within hospices is still to be developed.

Method Paramedics draw on a multitude of skill sets from symptom management, physical assessment, signposting, referrals and many more, and are adept at talking and communicating with people in the very worst of situations showing empathy and care, a baseline for all palliative care. My role as a paramedic in the hospice team is to conduct assessments, using my experience of visiting patients within the community and deciding on a course of action/referral or input required.

Outcomes Mountbatten Isle of Wight has seen two paramedics integrate well with the community palliative care team.
Paramedics have been able to give IV infusions to patients within their homes to help avoid clinical environments. Paramedics have extensive patient assessment and diagnostic skillsets and these have been added to by in-house training on catheterisation/advance care planning and end-of-life care supplementing the care provided by nursing staff within the community team.

Conclusion The role of the paramedic within hospice community teams is still in its infancy. We know that hospice services work differently across the country and therefore there are no hard and fast rules for what a paramedic may do.

\section{P-124 FROM HOSPICE VOLUNTEER TO COMPASSIONATE CITIZEN: SHIFTING THE BALANCE OF CARE AND POWER}

'Lynn Darke, 'Maggie Young, 'Anna Lloyd, 1,2Erna Haraldsdottir. 'St Columba's Hospice Care, Edinburgh, UK; ${ }^{2}$ Queen Margaret University, Edinburgh, UK

\subsection{6/spcare-2021-Hospice. 141}

Background Compassionate Neighbours, originated from the Public Health Palliative Care model, is a community development project aimed at developing mutually beneficial relationships that also helps build social capital and capacity within our communities. One hospice located in Scotland developed such an approach by expanding the role of volunteers within the hospice. Whilst hospices have long valued volunteers for their support in the provision of a de-medicalised, holistic approach to care, this new initiative reflects a longer-term, citizen-led investment that can equip communities to help one another during times of increased health need, loss and grief. Initiated and sustained during the COVID-19 pandemic, the project used an online blend of discussion, reflection and learning before matching Compassionate Neighbours with local people known to the hospice.

Aim To capture 'compassionate neighbours' experience and the impact of transitioning from a traditional volunteering role to a role reflecting the ethos of compassionate citizen.

Method Focus group inclusive of 10 volunteers that were part of the compassionate communities initiative and interviews with six people in the community they were 'matched with'.

Results Analysis of the data so far has highlighted how:

- The partnering process enabled the building of reciprocal relationships between volunteers and those they were matched with; vital to the success of the initiative.

- The Compassionate Neighbours negotiated, developed and sustained a mutually beneficial relationship with those they were matched with, reflecting the compassionate communities approach within the Public Health Palliative Care model.

Conclusion Central to the project's early success and future growth has been the emergence of a different kind of volunteer; one who sees him or herself as part of a community-led social movement involving ordinary people capable of responding in practical and compassionate ways to shared problems and needs in their own communities.

\section{P-125 THE CREATION OF HOSPICE OUTREACH DISCHARGE SUPPORT (HODS) TEAM}

Kate Martin. Nottinghamshire Hospice, Nottingham, UK

10.1136/spcare-2021-Hospice. 142 
Background Nottinghamshire Hospice's day therapy unit closed its doors to patients during the COVID-19 pandemic. Government advice was supporting discharge from hospital wherever possible (NHS England and NHS Improvement, 2020). When asked, more than four in five people say they would prefer to die at home (Hoare, Morris, Kelly, et al., 2015). Patients receiving care from specialist palliative care teams tend to do better than those without (Higginson \& Evans, 2010).

Aims To expedite the discharge of patients at end-of-life from hospital and prevent unnecessary hospital admission for those in the community so that more people achieve their preferred place of death (PPD) with the provision of a dedicated palliative care service.

Methods March-May 2020: consultations with fast track continuing care (FTCC), model planning, internal consultation, workforce transformation. May-July 2020: recruitment, service launch. July-March 2021: continuous service provision. We worked closely alongside FTCC to support patients either in hospital that wished to come home but needed a package of care or, patients that were deteriorating at home and required end-of-life care. An initial assessment by a registered nurse from the Hospice Outreach Discharge Support (HODS) team meant more timely referral into extended palliative community services including provision of specialist equipment and access to other hospice services e.g. bereavement support. It also focuses on the completion of EPaCCS and ReSPECT forms identifying PPD.

Results Number of patients treated by HODS May 20 March $21=195$.

$64 \%$ (124) died at home.

$23 \%$ (44) remained well enough to be discharged to domiciliary care agency.

$10 \%$ (20) admitted to hospital.

$3 \%$ (6) admitted to nursing home.

Average length of stay on HODS $=10$ days

PPD achieved $=95.87 \%[4]$

Conclusion The creation of HODS allows more people at end-of-life to remain in their PPD and with greater access to support services preventing carer burnout and unnecessary admission to hospital.

\section{P-126 HOSPICE@HOME TO PALLIATIVE RESPONSE TEAM}

Humaira Jamal, Elizabeth Kennedy. Peace Hospice, Watford, UK

10.1136/spcare-2021-Hospice. 143

\section{Background}

- Our Hospice at Home $(\mathrm{H} @ \mathrm{H})$ service supports 31 GP practices (population 315,000 patients).

- Pre- COVID-19 pandemic service provision of seven-days 9 am-5 pm was reviewed.

- Enhanced model developed supporting people wishing to die at home. (DIUPR local CCG 45.9 vs 46.6 England 2017 ONS).

- DIUPR increased during the pandemic.

\section{Aims}

- Increase ease of access to care provision at home through increased service hours.

- Increase medical support to develop a specialist palliative care virtual beds model.
- Uphold hospice's strategic aims and values of 'widening access' and 'reaching more people'.

- Uphold 'Ambitions for Palliative Care' - 'each person gets fair access to care' (National Palliative and End of Life Care Partnership, 2015).

Methods

- Collaborative appointment (Hospice and NHS) of new community palliative consultant.

- Use of triage priority rating.

- Agreed a rapid service pathway for those unstable/dying.

- New service launched September 2020 with increased hours ( 8 am to $10 \mathrm{pm}$ ) and ability to review patients same day.

- Supported out of hours by on-call palliative consultants.

- Virtual beds model supported by daily 'ward rounds' and updated plan of care.

- Data collection - number of referrals, referral source, diagnosis, place of death.

Results

\begin{tabular}{ll}
\hline & Nov 20-May 21 \\
\hline Referrals & 362 \\
Patients seen & 144 \\
Died & 166 \\
Discharged & 221 \\
Death Location\% & \\
Hospital & $4 \%$ \\
Patient's own home & $80 \%$ \\
Hospice & $11 \%$ \\
Care Home & $4 \%$ \\
\hline
\end{tabular}

Conclusion Service activity increased. $84 \%$ of patients died in their usual place of residence. Daily review of patient plans enabled continuity of care. Clear contact information required for patients and families as different service involvement can be confusing. Referral process between partner organisations needed re-discussion and review.

Future planning

- Priority ratings to be reviewed.

- Strengthen relationships with GP practices by attending Gold Standards Framework meetings.

- Increase use of data collection including user feedback to support the development of service to enable more patients to be reached in line with identified growing demand.

\section{P-127 A POPULATION HEALTH MANAGEMENT APPROACH TO END-OF-LIFE CARE: INCREASED PANDEMIC RESILIENCE}

Julie Barker. Nottingham and Nottinghamshire ICS, Nottingham, UK

10.1136/spcare-2021-Hospice. 144

Aims To meet the needs of the increasingly aged frail population (projected to almost double by 2050), an integrated population-based end-of-life care service, founded on Gold Standards Framework (GSF) principles and the Ambitions Framework is using a collaborative approach to learn lessons, address inequity and build on its solid foundations.

Methods Mid-Notts End of Life Care Together Alliance (EOLCT) spread GSF to GP practices and care homes, 